Jérôme Baschet, La civilisation féodale : de l'an mil à la colonisation de l'Amérique

Paris, Aubier, 2004, 565 p. (bibliogr., index, illustr., cartes) (« Collection historique »)

\title{
Catherine Vincent
}

\section{(2) OpenEdition}

Journals

Édition électronique

URL : http://journals.openedition.org/assr/2502

DOI : 10.4000/assr.2502

ISSN : $1777-5825$

Éditeur

Éditions de l'EHESS

Édition imprimée

Date de publication : 1 octobre 2004

Pagination : 53-158

ISBN : 2-222-96754-6

ISSN : 0335-5985

Référence électronique

Catherine Vincent, " Jérôme Baschet, La civilisation féodale : de l'an mil à la colonisation de l'Amérique », Archives de sciences sociales des religions [En ligne], 128 | octobre - décembre 2004, document 128.48, mis en ligne le 18 novembre 2005, consulté le 22 septembre 2020. URL : http:// journals.openedition.org/assr/2502 ; DOI : https://doi.org/10.4000/assr.2502 
produits, et d'en souligner la dimension paradoxale et ambivalente. C'est en effet, estime R. B., dans une économie morale, celle du clientélisme et plus généralement de la «politique du ventre », qui, a priori, a peu d'affinités électives avec le modèle démocratique, que la «domestication de la modernité démocratique »s'opère.

L'analyse de la démocratisation béninoise et des mutations des imaginaires politiques ne peut pas faire abstraction du religieux : il en est donc logiquement question dans cette étude. Couvrant la période 1989-1996, de l'effondrement du régime révolutionnaire de Mathieu Kérékou jusqu'au retour au pouvoir, par la voie des urnes, de celui-ci, désormais reconverti en démocrate et en chrétien, l'ouvrage n'aborde pas les effets politiques importants de cette conversion au christianisme du chef de l'État. Dans la première partie, l'A. consacre quelques pages au rôle du clergé et à celui d'intellectuels catholiques dans les mobilisations de la fin des années 1980 ainsi que dans l'organisation et le déroulement de la Conférence nationale de 1990. Il montre aussi comment le religieux, en particulier le vodun, et l'occulte, à travers les accusations de sorcellerie, informent les représentations et les répertoires d'action collective durant la phase de contestation et de revendication pluraliste. Il consacre surtout, dans la dernière partie, des développements plus longs d'une part à la revitalisation des cultes vodun consécutive à la libéralisation politique et, d'autre part, aux représentations de l'invisible en contexte démocratique.

Après avoir rappelé l'ambivalence de l'attitude du régime révolutionnaire à l'égard des cultes traditionnels, R. B. interprète la réactivation des cultes vodun depuis 1990, au même titre que le renouveau des structures monarchiques, comme significative de «procédures d'énonciation de la démocratie », traduisant, par des innovations institutionnelles ou par la modernisation des pratiques et des discours religieux, un repositionnement dans l'espace public et une appropriation du changement politique. Le registre de l'invisible constitue tout autant une instance d'adaptation aux changements et un vecteur paradoxal de domestication démocratique. Étudiant, à partir d'entretiens réalisés auprès de Béninois, les transformations dans « l'économie morale du pouvoir légitime » et les glissements dans les imaginaires politiques, R.B. relève un sensible apprivoisement de la «puissance » des détenteurs de l'autorité, lesquels apparaissent moins dépendants des forces de la nuit et moins effrayants. Si le recours aux forces occultes apparaît toujours, dans les représentations populaires, nécessaire pour conquérir et garder le pouvoir, il n'est cependant plus suffisant.
D'autres critères, comme le succès électoral ou la responsabilité politique, sont aussi avancés par les personnes interrogées.

Dans cette réflexion sur les rapports entre culture et changement politique, R.B. insiste sur l'ambivalence des vertus civiques qui émergent et sur les paradoxes de la consolidation démocratique au Bénin. Dans une telle perspective, le plus souvent convaincante sous la plume de 1'A., au style parfois légèrement redondant, le religieux et l'occulte, par leur pertinence sociale et leur plasticité évidente, constituent effectivement, parmi d'autres, des vecteurs importants de ces transformations ambiguës.

Cédric Mayrargue.

BASCHET (Jérôme).

La Civilisation féodale : de l'an mil à la colonisation de l'Amérique. Paris, Aubier, 2004, 565 p. (bibliogr., index, illustr., cartes) (« Collection historique $»)$,

Dans ce gros volume, J.B. livre une synthèse très personnelle de l'histoire de l'Occident médiéval durant sa phase qualifiée de «féodale »: un livre informé au meilleur des travaux récents, qui fourmille d'idées, dont il ne saurait être question de dresser ici un compte rendu détaillé... Les lecteurs des Archives de Sciences Sociales des Religions y trouveront largement leur intérêt tant est prégnante, dans cette histoire, la matrice chrétienne, le rôle de l'Église latine et les pratiques religieuses dans leur évolution.

La réflexion se construit à partir de l'interrogation suivante, sans doute d'autant plus présente à l'esprit de l'auteur qu'il enseigne non seulement à l'École des Hautes Études en Sciences Sociales mais aussi, désormais, au Mexique : comment expliquer le destin singulier de l'Occident dans l'histoire humaine et rendre compte de la force expansive qui le poussa vers les rivages du Nouveau Monde, notamment, et lui permit d'instaurer la domination que l'on connaît ? Dans ce processus, sur lequel bien des devanciers se sont penchés, l'A. entend rendre aux cinq siècles qui vont de l'an mil à la découverte de l'Amérique le rôle majeur qui fut le leur, pour en finir définitivement avec la sombre image qui s'y attache traditionnellement, en contraste avec les temps modernes, baptisés de manière éloquente temps des Lumières...

C'est ainsi que J.B. construit sa vision du « système féodal » qui, pour lui, a constitué le moteur de l'expansion occidentale : «Si l'Europe se lance à l'assaut du monde à partir du $\mathrm{XVI}^{\mathrm{e}}$ siècle, ce n'est pas parce qu'elle aurait 
inventé le capitalisme, mais bien d'abord parce qu'elle a inventé le féodalisme» (p. 528). Ce "féodalisme» (une appellation qui pourra surprendre) repose, selon 1'A., sur deux grands principes. Le premier est l'instauration d'une domination locale pesante, «presque totale », mais équilibrée et efficace. Le second réside dans le système ecclésial, qualifié d'institution dominante du féodalisme, auquel son «art d'articuler les contraires » et sa « rigueur ambivalente » ont conféré un extraordinaire caractère dynamique. Telle est, sommairement résumée, la thèse que défend brillamment l'A. et qui le conduit, après une première partie qui rappelle la formation et l'essor de la chrétienté médiévale, à mettre en valeur en quoi l'institution ecclésiale a donné sa forme même au système féodal, en passant en revue les cadres temporels et spatiaux, la logique du salut, la vision de l'homme, les structures de parenté et l'expansion des images, un domaine qui lui est cher.

Loin d'une image faite d'obscurantisme et de stagnation, l'A. renvoie de l'époque médiévale un portrait qui, sans angélisme, insiste sur son puissant dynamisme interne. Ce dernier serait donc largement redevable à l' « institution ecclésiale » telle qu'elle a été construite à partir de la réforme grégorienne, en Occident. On ne saurait cependant confondre cette dernière, incarnation historique d'une vision précisément datée du christianisme, avec la phénoménologie de cette religion. Or on a trop souvent l'impression, en lisant ces pages, que le christianisme et l'Église occidentale se confondent et sont enfermés dans leurs réalités médiévales... On remarquera en outre que l'expansion lointaine de l'Occident, dont on est loin de nier les racines anciennes qui plongent dans la phase d'expansion des $\mathrm{XI}^{\mathrm{e}}$ $\mathrm{XIII}^{\mathrm{e}}$ siècles, démarre à une époque où la belle construction unitaire fondée sur l'Église romaine tend à voler en éclat sous les coups de boutoir des États naissants. Rendre justice à ce que le destin de l'Occident doit au christianisme ne saurait se contester; mais dans la mesure où ce dernier ne se borne pas à ses modalités occidentales, reste encore ouverte la question initiale posée par l'ouvrage quant aux origines de la spécificité de l'aventure européenne...

Catherine Vincent.

128.49

BAUER (Olivier).

Les Rites protestants en Polynésie française. “Quand faire, c'est dire”. Paris, L'Harmattan, 2003, 288 p. (préface de Pierre Centlivres,) (bibliogr.).

Tiré d'une thèse de théologie soutenue à l'université de Lausanne en 1999, cet ouvrage se propose d'analyser les stratégies de ritualisation au sein de l'Église évangélique de Polynésie française à travers la description de quatre cérémonies religieuses : le culte, les funérailles, la confirmation et la cène, et ce en privilégiant une approche sensitive - voir, entendre, toucher, sentir, goûter.

L'auteur, pasteur aumônier suisse envoyé dans le cadre de la CEVAA (communauté évangélique d'action apostolique) à Tahiti de 1993 à 1999 fournit une multitude d'informations et de descriptions qui donnent le point de vue de quelqu'un qui est tout à la fois à l'intérieur - en tant que pasteur - et à l'extérieur - culturellement - de l'Église évangélique de Polynésie française. Il qualifie sa démarche de «théologie pratique culturelle» et se demande comment " comprendre le rapport entre l'universalité de l'Évangile et son inscription dans des contextes particuliers » (p. 23), notamment la façon dont s'élabore et se diffuse une théologie contextuelle qui tient compte des cultures polynésiennes.

Après une première partie consacrée à la présentation générale de la Polynésie française, 1'A. s'attache dans une seconde partie à l'élaboration d'un cadre théorique en rappelant différentes approches des rites (Goffman, Bourdieu, l'école de Palo Alto) et en reprenant les travaux de Catherine Bell. Celle-ci souligne que « ritual communicates by "modeling": strictly speaking, it does not send messages but creates situations » (Ritual Theory, Ritual Practice, New York, Oxford University Press, 1992, p. 111) et préfère utiliser le terme de ritualisation dans la mesure où ce concept introduit une dynamique dans l'approche des rites. C'est un des mérites de l'ouvrage de montrer que les rites étudiés évoluent, se transforment et sont l'objet d'interprétations différentes et parfois antagonistes. C'est le cas tout particulièrement, dans la troisième partie de l'ouvrage, de la confirmation du baptême qui, selon les avis, devrait être réservée aux personnes d'âge mûr, mariées et menant une vie respectable ou au contraire s'adresser à des paroissiens jeunes et non mariés qui transforment la confirmation en "rite de passage" autorisant, aux yeux de la communauté paroissiale, leur entrée dans la vie adulte.

On peut néanmoins s'interroger sur le bien fondé de la démarche choisie, l'approche par les sens, qui laisserait de côté l'analyse des discours (prédications, lectures bibliques, chants) au motif qu' « en examinant les sensations que les rites font éprouver, j'ai l'impression de m'être plus approché de la vérité des rites, que si je m'étais cantonné au discours théologique officiel qui les justifie et les interprète » (p. 251). Pourtant l'A. définit préalablement le rite 